\title{
Antipsychotic Polypharmacy for the Management of Schizophrenia: Evidence and Recommendations
}

\author{
Markku Lähteenvuo $^{1}$ (D) Jari Tiihonen ${ }^{1,2,3}$ (D)
}

Accepted: 7 June 2021 / Published online: 1 July 2021

(c) The Author(s) 2021

\begin{abstract}
Schizophrenia is a debilitating illness with a lifetime prevalence estimate of $0.6 \%$ and consists of symptoms from the positive, negative, and cognitive domains. Social support, therapy, psychoeducation, and overall case management are very important aspects of the treatment of schizophrenia. However, as abnormalities in neurotransmission are one of the key findings of schizophrenia pathology, pharmacotherapies are cornerstones of the management of schizophrenia. Antipsychotics have been used as the primary pharmacological treatment of schizophrenia. These agents often have a good effect on reducing positive symptoms, but may not markedly improve negative symptoms or cognitive defects. However, at least $20 \%$ of individuals with schizophrenia do not experience a substantial response from monotherapy with antipsychotics. Further, despite evolving treatment protocols and advances in early recognition of the disorder, $70 \%$ of patients with schizophrenia require long-term, even lifetime, medication to control their symptoms and do not achieve complete recovery. To address these shortcomings, clinicians and research scientists have explored different combinations of treatments, polypharmacy, to improve the treatment of patients. Antipsychotic polypharmacy has been shown to cause more side effects than monotherapy, which is the main reason why most treatment guidelines caution against it. Antipsychotic monotherapy should be strived for and clozapine should be tried at the latest if two monotherapy trials with other antipsychotics have failed and no absolute contraindications exist. If residual symptoms exist despite trials of adequate dose and duration, other reasons that may reduce treatment effect should be ruled out. Long-acting injectables or blood concentration measurements should be considered to affirm compliance and proper serum levels. Antipsychotic polypharmacy should be considered and discussed with patients from whom the aforementioned procedures do not produce a satisfactory treatment result. In some cases, antipsychotic polypharmacy may produce better results than other forms of treatment augmentation, such as benzodiazepines. In particular, combining aripiprazole with clozapine may be effective in reducing treatment side effects or residual symptoms, and this is likely to hold true for combining other partial dopamine $\mathrm{D}_{2}$ agonists with clozapine as well, although currently scant data exist. More research is needed, both in controlled but also real-world settings, to define optimal antipsychotic polypharmacy and/or other psychotropic treatment augmentation strategies for specific patient groups and situations.
\end{abstract}

\section{Key Points}

Antipsychotic polypharmacy is frequently used, although not recommended by treatment guidelines

Markku Lähteenvuo

Markku.Lahteenvuo@niuva.fi

1 Department of Forensic Psychiatry, Niuvanniemi Hospital, University of Eastern Finland, Niuvankuja 65, 70240 Kuopio, Finland

2 Department of Clinical Neuroscience, Karolinska Institutet, Stockholm, Sweden

3 Center for Psychiatry Research, Stockholm City Council, Stockholm, Sweden

Antipsychotic polypharmacy is effective and may be applicable in certain clinical situations where monotherapy with non-clozapine antipsychotics and clozapine have failed or clozapine is contraindicated

Antipsychotic polypharmacy does not seem to increase mortality but may increase the prevalence of treatmentrelated side effects, although some combinations may also reduce side effects 


\section{Schizophrenia}

Schizophrenia is a serious mental disorder with a median lifetime prevalence estimate of around $0.6 \%$, with some population-specific variation [1]. It has been thought to be a neurodevelopmental disorder [2], although the specific triggers for the development of the disorder are still somewhat unknown. Genetics play an important role at least in terms of risk, as schizophrenia has been shown to be highly heritable with estimates at around 80\% [3]. Some genes with a high impact have been discovered, although the risk is likely carried by multiple genes with smaller individual effects $[4,5]$. However, genetics are not the be-all and endall, as environmental factors, such as pregnancy and birth complications, childhood trauma, migration, social isolation, urbanicity, and substance abuse, also play a major role in whom the disorder manifests [6]. This is evident as monozygotic (identical) twins only have about $40-50 \%$ concordance rates for schizophrenia [7]. As the risk factors for schizophrenia are diverse, it is likely that schizophrenia is not a single entity always stemming from the same root cause, but rather a multifactorial disorder, which may be somewhat distinct between different individuals [8], or even different sexes [9]. As the clinical symptoms seen in individuals are rather similar, although different subtypes of schizophrenia have been classified, it is likely that these possibly distinct etiological processes converge at some point to cause disturbances in specific neurobiological systems. The systems most often implicated in schizophrenia pathophysiology revolve around the neurotransmitters dopamine and glutamate [10].

\subsection{Basis of Treatment}

Cardiologists have a saying: "Time is myocardium". An equivalent saying for psychiatrists treating schizophrenia could be: "Time is cognition". Evidence is emerging, although still very limited, that early (pre-psychosis) effective treatment of schizophrenia is vital in preserving patients' cognition and ability to function $[11,12]$. Social support, therapy, psychoeducation, and overall case management are very important aspects of the treatment of schizophrenia in all stages of the disorder [13-15]. However, as changes in neurotransmitter levels are one of the key findings of schizophrenia pathology, pharmacotherapies are thought to be one of the most important cornerstones of the management of schizophrenia [10]. Schizophrenia causes symptoms in different domains, often classified as positive symptoms, negative symptoms, and cognitive symptoms. Positive symptoms are classified as symptoms in the domain that are present in excess in individuals with schizophrenia as compared with individuals without schizophrenia and include hallucinations, delusions, and disorganized behavior. Negative symptoms are symptoms in domains in which individuals without schizophrenia function normally but individuals with schizophrenia have reduced functioning, expressed as increased apathy, avolition, and anhedonia that may lead to isolation or self-neglect. Cognitive symptoms include impairment of different cognitive functions, such as problems with executive functioning, information processing, and attention. [14, 15] The symptoms are most often assessed with the Positive and Negative Syndrome Scale, where lower numbers indicate fewer symptoms [16].

Antipsychotics (agents working as dopamine $\mathrm{D}_{2}$-receptor antagonists or partial agonists, in addition to having other receptor affinities) have been used as the primary pharmacological treatment of schizophrenia [15]. Some $34 \%$ of patients with schizophrenia do not respond to non-clozapine antipsychotics and are deemed treatment resistant [8]. These patients are treated with clozapine, which is also classified as an antipsychotic, although it may exert its effects through mechanisms other than $\mathrm{D}_{2}$-receptor antagonism [17]. These agents often have a good effect on reducing positive symptoms, but may not markedly improve negative symptoms or cognitive defects [15]. However, at least $20 \%$ of individuals with schizophrenia do not receive a clear benefit from monotherapy with antipsychotics $[8,18,19]$. This may be due to differences in disorder etiologies as stated above, but may also be due to differences in other individual factors, such as metabolism speed, substance abuse, or medication adherence. Further, despite evolving treatment protocols and advances in early recognition of the disorder, $70 \%$ of patients with schizophrenia require long-term, even lifetime, medication to control their symptoms and do not achieve complete recovery [20]. To address these shortcomings, clinicians and research scientists have explored different combinations of treatments, polypharmacy, to improve the treatment of patients. It is possible that trying to 'cure' fully manifested schizophrenia is futile, especially with antipsychotics, and that treatment may need to be initiated at a very early stage, possibly with very different medications, such as anti-inflammatory medications [21-23]. However, it does not diminish the fact that there currently exists a large pool (up to $20 \%$ ) of patients with insufficient treatment response from antipsychotic monotherapy (APM) [8]. 


\section{Antipsychotic Polypharmacy (APP) and Treatment Guidelines}

This review focuses on the use of antipsychotic polypharmacy (APP) in the treatment of schizophrenia, defined as the concurrent use of any two antipsychotic medications. Several meta-analyses of randomized clinical trials (RCTs) have been conducted to evaluate the feasibility of APP, but the results have been mixed, possibly owing to small sample sizes and a lack of separation between low-quality and highquality studies in the analyses [24-28]. Many high-quality reviews on APP have been written during the years, with older reviews mostly supporting monotherapy, but newer reviews also acknowledging the feasibility of APP in certain situations, such as for clozapine-resistant patients [25, 26, 29-37]. However, most guidelines still have rather categorical statements against APP. This likely stems from the fact that most guidelines are based on RCTs, which have limited possibilities for long-term follow-up and exploring maintenance treatments, and are often more focused on acute-phase treatment. Randomized controlled trials also often have very stringent inclusion criteria, which have become more stringent over the years, leading to the exclusion of many patient groups, including those with schizophrenia that is resistant to APM treatment [38-41]. This review focuses on the more recent data based on large studies emphasizing real-world data and high-quality meta-analyses.

The very recent guidelines of the American Psychiatric Association on the treatment of schizophrenia endorse monotherapy and do not acknowledge situations in which APP would be recommended. This is to be expected, as the guideline is based on a review by McDonagh et al. [42], which mainly focused on APM.

The National Institute for Health and Care Excellence guideline: "Psychosis and schizophrenia in adults: prevention and management" published in 2014 and reviewed in 2019 guides against using regular combined antipsychotic medication, except for short periods (e.g., when changing medication). However, the guideline does allow for adding an additional antipsychotic to augment clozapine treatment, if clozapine monotherapy has proven ineffective. In this case, the guideline recommends selecting a drug that does not compound the common side effects of clozapine [43].

The World Federation of Societies of Biological Psychiatry guidelines for biological treatment of schizophrenia recommend that APP should only be considered in certain individual cases such as patients with treatment-resistant schizophrenia. They state that the combination of clozapine with another second-generation antipsychotic (possibly risperidone) might have some advantages compared with monotherapy [44]. Otherwise monotherapy should be used.

The Finnish Current Care Guideline for schizophrenia (3/2020, https://www.kaypahoito.fi/hoi35050 [45]) recommends APM. However, it states that some patients may benefit from the concurrent use of two antipsychotic medications and that combining aripiprazole with another antipsychotic may reduce negative symptoms.

Although treatment guidelines mainly recommend APM, APP is widely used in clinical practice. Antipsychotic polypharmacy has been estimated to be used in $10-20 \%$ of outpatients with schizophrenia and up to $40 \%$ of inpatients with schizophrenia, although differences between different parts of the world naturally exist. A fairly recent systematic review found time-dependent and country-dependent trends in the prevalence of APP, with a median APP rate of $19.6 \%$ (note: this study included $17 \%$ of patients without a schizophrenia diagnosis) [46]. Antipsychotic polypharmacy was least prominent in North America (16\%) and Oceania (16.4\%) and more prevalent in Europe (23\%) and Asia (32\%), although the recent REAP survey study has reported numbers as high as $42.6 \%$ for Asia [47]. A recent, large, real-world, evidence nationwide cohort study by our group $(n=62,250)$ showed that up to $57.5 \%$ of Finnish patients with schizophrenia were receiving APP for at least 90 days during their follow-up (median follow-up time 14.1 years), although some of this time is likely to have been antipsychotic cross-titration [48]. The use of two concurrent antipsychotics has been reported to be the most prevalent number of concurrent antipsychotics when using APP, but a study on Turkish outpatients $(n=280)$ reported that some patients are prescribed up to five concurrent antipsychotics (29\% receiving monotherapy, $44.4 \%$ with two antipsychotics, $24.4 \%$ with three, $1.4 \%$ with four, and $0.7 \%$ [two patients] with five) [49]. In general, the APP rate has been shown to correlate with inpatient status, use of first-generation antipsychotics and anticholinergics, less antidepressant use, and greater use of long-acting injectable formulations of antipsychotics [46].

\section{APP: Reasons}

Clinical reasons to resort to APP may be as wide and varied as the prescriber base, although the most often cited reason is insufficient treatment response on positive symptoms with APM [50]. Other reasons include reducing negative symptoms, decreasing the dose of any one medication, reducing medication costs, and reducing extrapyramidal symptoms [50,51]. Antipsychotic polypharmacy may also be initiated to target specific comorbid symptoms, such as anxiety, cognitive dysfunction, impulsive/violent behavior, 
and sleep disturbances, instead of other medications used for these symptoms. Prescription of additional sedating antipsychotics for sleep disorders instead of benzodiazepines is sometimes used, and may lead to better results [52], especially considering the negative effects of longlasting benzodiazepine use on suicide risk, cognition, concentration, and anxiety [53-55]. Predictors of long-term APP have been shown to include previous treatment with clozapine and long-acting injectables, previous treatment with APP, the symptom severity of positive symptoms, increased service utilization, social factors, and sociodemographic status (younger age, being single) [56-58].

\section{APP: Evidence Supporting or Neutral Towards Use}

\subsection{Clinical Trials}

Several publications have reported the benefits of APP. A recent review, meta-analysis, and meta-regression of RCTs adding another antipsychotic to APM vs continuing with monotherapy found that antipsychotic augmentation was superior to monotherapy regarding total symptom reduction with a good effect size (16 studies, $N=694$, standardized mean difference $-0.53,95 \%$ confidence interval [CI] -0.87 to $-0.19, p=0.002$ ), although this was only apparent in open-label low-quality trials $(p<0.001)$, but not in double-blinded $(p=0.12)$ or otherwise high-quality trials $(p=0.226)$ [28]. Additionally, no significant differences were observed when using study-defined multiple response rates criteria, although such differences were noted in their previous meta-analysis with a smaller sample [24]. No differences in efficacy were observed regarding discontinuation, global clinical impression or positive, general, and depressive symptoms, although negative symptoms improved with aripiprazole augmentation (eight studies, $N=532$, standardized mean difference $-0.41,95 \%$ CI -0.79 to $-0.03, p=0.036$ ). Adverse effects were few, with $\mathrm{D}_{2}$-receptor antagonist augmentation even associated with less insomnia and aripiprazole augmentation with reduced prolactin levels and body weight.

Another meta-analysis of 42 antipsychotic combinations or augmentations in the treatment of schizophrenia did not find clear evidence to recommend the use of APP over APM, although the authors did note beneficial signals for some APP and augmentation strategies. However, the effect sizes of the treatments were inversely correlated with the quality of the studies included, cautioning against making affirmative conclusions on these data [59].

Several studies have also looked at how patients fare when they are transitioned from APP to monotherapy. A systematic review and meta-analysis by Matsui et al. [60] of six RCTs involving a total of 341 patients found that switching from two antipsychotics to monotherapy was associated with a significant risk of treatment discontinuation (risk ratio $2.28,95 \%$ CI $1.50-3.46$ ). No significant differences were observed in relapse rates, psychopathology, neurocognition, extrapyramidal symptoms, or body weight between the two groups. They caution interpretation, as the overall quality of evidence in the studies was low to very low. Another issue to consider in these types of studies, as highlighted by Baandrup in a commentary, is the dosing [61]. Unless the doses of the agent remaining as monotherapy are increased to compensate for the reduction in the other antipsychotic, any results in effectiveness will be in favor of the APP group, as members of that group will have higher combined total doses. Similarly, the amount of side effects would be expected to be in favor of the monotherapy group, considering the lower total doses. Despite this, in individual studies switching from APP to monotherapy has been largely successful, although there seems to exist a population of patients who do not tolerate switching to monotherapy. In a study conducted in the USA, 127 outpatients with schizophrenia across 19 sites were randomized to stay on APP or switch to monotherapy. The trial lasted for 6 months, with a 6-month naturalistic follow-up. Individuals assigned to switch to monotherapy had shorter times to all-cause treatment discontinuation; while around two thirds of the patients switched to monotherapy were able to continue in that group without differences in psychiatric symptomatology or the amount of hospitalizations, a third of the patients had to be returned to APP. The monotherapy group also lost weight as compared with the APP group. Similar results were observed in a smaller and shorter study by Borlido et al., who observed that almost $80 \%$ of patients with schizophrenia or schizoaffective disorder $(n=35)$ could safely switch from APP to monotherapy without indication of clinical worsening as measured by the Clinical Global Impression Scale or the Brief Psychiatric Rating Scale, but 20\% could not. Constantine et al. organized a similar switch study with 104 adult outpatient participants with schizophrenia [62]. The participants were followed for 1 year with assessments every 2 months. In this study, participants who switched from APP to monotherapy experienced greater increases in symptoms, which manifested in the second half of the follow-up time, and greater rates of discontinuation (42\% in the switch arm vs $13 \%$ in the APP arm). They also performed a follow-up study that assigned 99 adult participants with schizophrenia or schizoaffective disorder to either stay on APP or switch to monotherapy, again followed for a year [63]. This time, half of the participants in both groups were receiving combinations involving either clozapine or longacting injectables and the other half combinations of oral non-clozapine antipsychotics. They observed that patients 
who switched from the non-clozapine oral combination to monotherapy experienced significant increases in symptoms, but also benefits in side effects, as compared with participants who remained on similar APP. No differences were observed for patients switching from clozapine or longacting injectable APP combinations to monotherapy, either in symptoms or side effects. They concluded that patients treated with clozapine or long-acting injectable APP can be safely switched to similar monotherapy, while there are risks associated with switching patients from non-clozapine non-injectable APP.

Antipsychotic polypharmacy may also lead to monetary savings if combinations of less expensive medications are used instead of larger doses of more expensive medications, as demonstrated in a study by Lin et al. [51]. They randomized 92 patients with schizophrenia into two groups of 46 individuals in a 6-week double-blind fixed-dose trial. One group received $800 \mathrm{mg} /$ day of amisulpride and the other group received $400 \mathrm{mg} /$ day of amisulpride combined with $800 \mathrm{mg} /$ day of sulpiride. Both groups were similar in terms of clinical characteristics at baseline, response rates (defined as a $30 \%$ reduction in the Positive and Negative Syndrome Scale total score), quality of life, and side effects, but the combination strategy was cheaper.

\subsection{Observational Studies}

A Hungarian nationwide population-based study investigated the comparative effectiveness of an APM switch vs antipsychotic augmentation (maximum two antipsychotics concurrently) in schizophrenia and other psychotic disorders [64]. They detected a significant overall advantage of polypharmacy over monotherapy for mortality and hospitalization, where both outcomes appeared more frequently during monotherapy (hazard ratio [HR] 1.62, 95\% CI 1.12-2.34 for mortality and HR 1.69, 95\% CI 1.43-1.99 for hospitalization), although monotherapy in general was associated with less treatment discontinuation. However, some combinations with depot formulations (excluding risperidone depot) were associated with less treatment discontinuation. Specifically, combining oral haloperidol with oral olanzapine was associated with significantly better outcomes than haloperidol monotherapy. All other statistically significant, beneficial polytherapy combinations were combinations of long-acting injectables with peroral medications. They took their findings to mean that APP may be superior during exacerbation of psychotic symptoms, but monotherapy may be superior for long-term sustained treatment.

A Japanese, 1-year duration naturalistic study examined the effectiveness of APM and APP in newly admitted patients with acute-phase schizophrenia $(n=1543)$ [65]. In this study, 581 of the patients did not respond to the first or second APM trial and were prescribed APP. Among the
581 patients, $89.8 \%$ showed an improvement on the Clinical Global Impression-Improvement Scale. The rates of side effects (hyperglycemia, prolactinemia, cholesterolemia, extrapyramidal symptoms, and QTc prolongation) were not higher in patients with APP and no serious adverse events were reported in the study.

We have also explored the effects of polypharmacy in two recent studies using a nationwide cohort from Finland $(n=62,250)$ and within-individual analysis models $[48,66]$. Our cohort included an even spread of male and female subjects $(50.2 \%$ vs $49.8 \%$, respectively) with a median age of 45.6 years. The median follow-up time was 14.1 years and $67.2 \%$ of the cohort used polypharmacy at some point during the follow-up (57.5\% for over 90 days). Our results showed that individuals were on average at a 7-13\% lower risk of psychiatric hospitalization when they were treated with APP instead of APM. The polytherapy combination associated with the lowest risk of psychiatric hospitalization was clozapine combined with aripiprazole (HR $0.86,95 \%$ CI $0.79-0.94$ as compared with clozapine monotherapy, HR 0.78 95\% CI 0.63-0.96 for first-episode patients). In general, clozapine monotherapy was the only monotherapy among the ten most effective treatments, all others were different forms of polytherapy including either clozapine or long-acting injectables. Antipsychotic polypharmacy was associated with a $9 \%$ reduced risk $(95 \%$ CI for HR 0.89-0.92) for all-cause hospitalization, with the clozapine plus aripiprazole combination being associated with the best outcome (22\% reduced risk, 95\% CI for HR 0.63-0.96) as compared with the best APM, clozapine. Antipsychotic polypharmacy was also associated with the lowest risk of somatic hospitalization (HR 0.79, 95\% CI 0.75-0.84 against no antipsychotic) and all-cause mortality (24\% reduced risk for death, 95\% CI 0.73-0.79, between-individuals analysis). [48] We also analyzed the dataset for somatic morbidity and mortality in a different manner in the second publication [66]. We compared different forms of monotherapies (both oral and long-acting injectables) and a category called 'polytherapy' (including all different forms of APP) against non-use of an antipsychotic. Analyzed this way, APP came in the top 50\% of specific treatments in every single analysis for somatic morbidity or mortality (fifth best position out of 20 for the risk of somatic hospitalization, $8 / 20$ for the risk of cardiovascular hospitalization, $4 / 20$ for the risk of all-cause mortality, $7 / 20$ for the risk of cardiovascular mortality, and $4 / 20$ for the risk of suicide mortality). From these studies, we concluded that certain types of APP may be feasible in the treatment of schizophrenia and the current treatment guidelines should modify their categorical recommendations discouraging the use of APP. [48]

We have also previously analyzed all-cause mortality with patients followed from their initial hospitalization in 
a nationwide, observational registry-based cohort study ( $n$ $=2588$, mean follow-up 4.2 years). In this study, APP (two or more concurrent antipsychotics) was not associated with increased mortality as compared to APM (HR 0.86, 95\% CI 0.51-1.44). In this study, use of other therapeutic groups was also studied. We found that use of antidepressants was not associated with a higher risk of suicide mortality, although use of benzodiazepines was related (HR 1.91, 95\% CI 1.13-3.22) [67].

We have also looked at the effectiveness of antipsychotics in preventing psychiatric rehospitalizations and treatment failure (psychiatric rehospitalization, suicide attempt, discontinuation, switch to other medication, or death) in a Swedish nationwide observational cohort study ( $n=29,823$, mean follow-up 5.7 years) [68]. During follow-up, $43.7 \%$ of the patients were re-hospitalized and $71.7 \%$ experienced treatment failure. In this study, APP was more effective than any non-clozapine oral APM, but less effective than clozapine or long-acting injections. Antipsychotic polypharmacy was associated with a $38 \%$ reduction in the risk for psychiatric rehospitalization (95\% CI 0.58-0.65 for HR), while the most effective treatment, long-acting paliperidone, was associated with a $49 \%$ reduction and the least effective treatment, oral flupentixol, with a non-significant $8 \%$ reduction. Antipsychotic polypharmacy was associated with a $39 \%$ reduction (95\% CI 0.57-0.64 for HR) in the risk for treatment failure as compared with oral olanzapine and came in second after clozapine monotherapy (HR 0.58, 95\% CI 0.53-0.63).

A study by Baandrup et al. explored whether the increased mortality from natural causes observed in patients with schizophrenia was associated with APP [69]. They used a population-based nested case-control design with 27,633 patients diagnosed with schizophrenia or other non-affective psychoses. Among the 193 deaths due to natural causes identified in the cohort they did not find an association of increased mortality in the APP group (odds ratio [OR] 0.91, 95\% CI 0.61-1.36 for two antipsychotics, OR 1.16, 95\% CI 0.68-2.00 for three or more antipsychotics). However, they did discover an increased risk of mortality with benzodiazepine augmentation (OR 1.78, 95\% CI 1.25-2.52).

A study from the UK looking at the effects of long-term APP ( $>6$ months) in 10,945 patients with a severe mental illness did not discover an increase in the overall risk of death or risk of death due to natural or unnatural causes for users of APP vs monotherapy in their fully adjusted models, although some analyses did hint at an increased risk [70]. Another UK study explored the association of use of APP with unplanned hospital admissions, emergency department visits, and mortality in a sample of 17,255 adults with serious mental illness. They found no significant associations with APP use as compared to monotherapy in increased mortality (HR 1.02, 95\% CI 0.76-1.37), unplanned hospital admissions (HR 1.14, 95\% CI 0.98-1.32), or emergency room visits (HR $0.95,95 \%$ CI 0.80-1.14) [71].

A Chinese, population-based nested case-control study included 157 patients with schizophrenia who had died of natural causes and 444 age-matched and sex-matched controls. The study looked at the risk of death due to natural causes in patients using AP monotherapy or APP as compared to patients not using antipsychotics. Both AP monotherapy (OR $0.27,95 \%$ CI $0.16-0.46$ ) and APP (OR 0.29, 95\% CI 0.12-0.70) were associated with comparable significantly reduced mortality due to natural causes [72].

\section{APP: Evidence Against Use}

Antipsychotic polypharmacy has been clinically associated with several disadvantages, such as increased prevalence and a higher severity of side effects, and reduced adherence, increased risk of medication interactions, and more medication errors because of treatment complexity [58, 73-75]. Patients receiving APP have also been shown to receive more prescriptions for other psychiatric comorbidities or medication-induced side effects of APP [58].

Although studies reviewed above have reported on a protective association with APP as compared with monotherapy on rehospitalizations, contrary evidence also exists. A recent study comparing APP (both clozapine and non-clozapine combinations separately) with clozapine monotherapy in the UK found that patients with a serious mental illness discharged from hospital with APP have a significantly increased risk of readmission (HR 1.4, 95\% CI 1.2-1.7) as compared with patients discharged on monotherapy [76]. This risk was even higher with patients discharged on clozapine APP (HR 1.8, 95\% CI 1.2-2.6), although an increased risk was not seen for patients on non-clozapine APP (HR 1.4, 95\% CI 0.9-1.9). The authors adjusted their model for several variables, but were not able fully to account for differences in initial disease severity or medication adherence during the follow-up. The APP group had patients with more hallucinations or delusions and more days of inpatient stay and outpatient contact during the previous 6 months. This study also included patients with bipolar disorder, who were more numerous in the monotherapy group (25.7\% in the monotherapy group and $14.4 \%$ in the APP group). This leads to the assumption that patients in the APP group had in general a more severe clinical manifestation, which may explain part of the increased risk observed.

The recent review, meta-analysis, and meta-regression by Galling et al. mentioned above showed $\mathrm{D}_{2}$ antagonist augmentation to be associated with more prolactin elevation than APM [28]. Indeed, a review of the safety and tolerability of APP by Gallego et al. has shown APP to be associated with an increased global side-effect burden, rates of Parkinsonian 
side effects, anticholinergic use, hyperprolactinemia, sexual dysfunction, hypersalivation, sedation/somnolence, cognitive impairment, and diabetes mellitus, although these results were based mostly on small and uncontrolled studies [29]. Effects on akathisia and mortality were inconclusive, and some beneficial effects of combining aripiprazole with an antipsychotic with a greater side-effect burden were noted, namely for reduced weight gain, dyslipidemia, hyperprolactinemia, and sexual dysfunction. If APP is to be used, guidelines recommend selecting antipsychotics with differing side-effect profiles. Although this approach may protect from the exacerbation of existing side effects, it may lead to a wider variety of side effects, unless the side effects are contrary to each other. Other variables may also lead to an increase in side effects. Drug-drug interactions, especially those arising from using drugs affecting the same metabolic pathways may have additive or reductive effects on plasma concentrations as well as the severity of side effects and adverse reactions, as has been shown for the combination of risperidone with levomepromazine, chlorprothixene, melperone, pipamperone, or prothipendyl [77-79]. Knowledge of cytochrome P450 (CYP) metabolizer status, especially for CYP2D6, may help predict these interactions.

Antipsychotic polypharmacy has been associated with detrimental effects on cognition, although these associations have been reported to be driven more by the antipsychotic daily dose, often higher in patients treated with APP, and thus not results from APP per se but rather the higher total dose [80]. However, some studies have not noted cognitive decline in patients treated with high-dose monotherapy or APP as compared to lower doses or monotherapy, indicating that polytherapy and higher doses may not be a causative factor for cognitive decline, but rather both cognitive decline and the need for more intensive treatment may be jointly mediated by other factors [81]. The data on the causal effects of APP on cognition are still somewhat conflicting.

\section{Considerations and Future Directions}

Antipsychotic monotherapy, either with clozapine or nonclozapine antipsychotics, should be strived for with most patients, as generally monotherapies incur less health service costs to society and give a lower overall risk for adverse effects [29, 82], although direct medication costs may be even higher to an individual patient [51]. Medication adherence may also be better with monotherapies, as the patient needs to remember to take only one antipsychotic [83]. Adherence has been shown to be better with long-acting injectables as compared with peroral medications and may be better with antipsychotics given as a single daily dose as opposed to several daily doses [83-85]. Antipsychotic polypharmacy may be appropriate for patients who still have symptoms despite treatment attempts with an adequate dose, an adequate choice of therapeutic, and confirmed adherence. An adequate dose may be affected by the metabolic status of the patient $[77,86]$. Slow metabolizers may experience side effects at lower doses and fast metabolizers may not achieve optimal treatment effects even with high doses. If the antipsychotic selected is known to be affected by CYP enzymes with marked genetic polymorphisms between individuals (such as CYP2D6), a pharmacogenetic panel or blood drug concentration measurement may be warranted before dose adequacy is determined [86, 87]. Furthermore, if used by the patient, the effect of other pharmaceuticals or over-the-counter products on the speed of metabolism and blood drug concentrations should be contemplated. Smoking status (e.g., clozapine and olanzapine), caffeine consumption (e.g., clozapine and olanzapine), eating schedule (e.g., lurasidone), and recreational drug use may also have an effect on blood drug concentrations [86]. In general, substance abuse may worsen compliance and adherence and may be a topic the patient is unwilling to face, unless a very trusting treatment relationship can be formed [88, 89].

Clozapine monotherapy is underutilized and should be considered for a wider spectrum of patients than it is currently used for [90-94]. Increasing clozapine use would also open up the door wider for combinations of clozapine with other antipsychotics and treatment augmentations, of which especially partial $\mathrm{D}_{2}$ agonists have produced very promising results $[48,95]$. Although these approaches still require a considerable research effort from the field to arrive at the most optimal combinations for specific situations, in general, these combinations seem to be well tolerated and no major barriers for research should exist. Clozapine, when properly used, is safe and usually well tolerated, and combining it with partial $\mathrm{D}_{2}$ agonists may allow a reduction in the clozapine dose and lead to reduced overall side effects [95-97].

Pharmacogenetics may offer a fast and cost-effective method to determine better optimized treatment approaches in the future, not only to plan initial monotherapy or sequential polypharmacy approaches, but also to determine that monotherapy trials have been properly dosed, especially if blood concentration measurements for the given compound do not exist or are laborious or expensive [86]. Initial pharmacogenetic studies were mostly small and the results modest $[98,99]$, but very promising signals from larger studies both for improved efficacy and tolerability when using pharmacogenetics have also arisen and pave the way for future research on this important topic [86, 87, 100]. In particular, research efforts in predicting good clozapine response, both in terms of efficacy and safety, are important and eagerly anticipated [101].

Even though APP may be a good choice for some patients, there is growing evidence that many patients currently on APP could be safely switched to APM $[62,63,102$, 
103]. Thus, whenever considering starting a patient on APP, the current symptomology and clinical status of the patient should be clearly recorded and a follow-up after initiation of APP is essential. If the patient does not improve, APP should be reverted back to monotherapy or other combinations explored. If the patient has improved and attained a stable condition, slowly and carefully reverting back to APM should be considered, as many patients seem to be able to tolerate this, and might benefit from APP only at times of symptom exacerbations [62, 102, 103]. This is also the case with patients for whom the symptomology before the initiation of APP is unclear or lacking proper documentation and the actual benefits incurred from APP remain unclear. Switching may be safer with patients taking clozapine or long-acting injectable APP [63]. However, if symptoms worsen during the switch to monotherapy, reverting back to APP is likely a better option.

\section{Conclusions and Recommendations}

Figure 1 presents a flowchart of the recommendations based on this review. Antipsychotic monotherapy should be strived for, especially for patients who achieve complete remission with it. Clozapine should be tried at the latest [92-94] if two monotherapy trials with other antipsychotics do not produce good results and no absolute contraindications exist. If residual symptoms exist despite adequate dose trials of adequate duration, other reasons that may reduce the treatment effect should be ruled out. Long-acting injectables or blood concentration measurements should be considered to affirm compliance and proper serum levels, even when no obvious signs of non-adherence are present if residual symptoms persist. Antipsychotic polypharmacy should be considered and discussed with patients from whom the previous recommendations do not produce a satisfactory treatment result. In

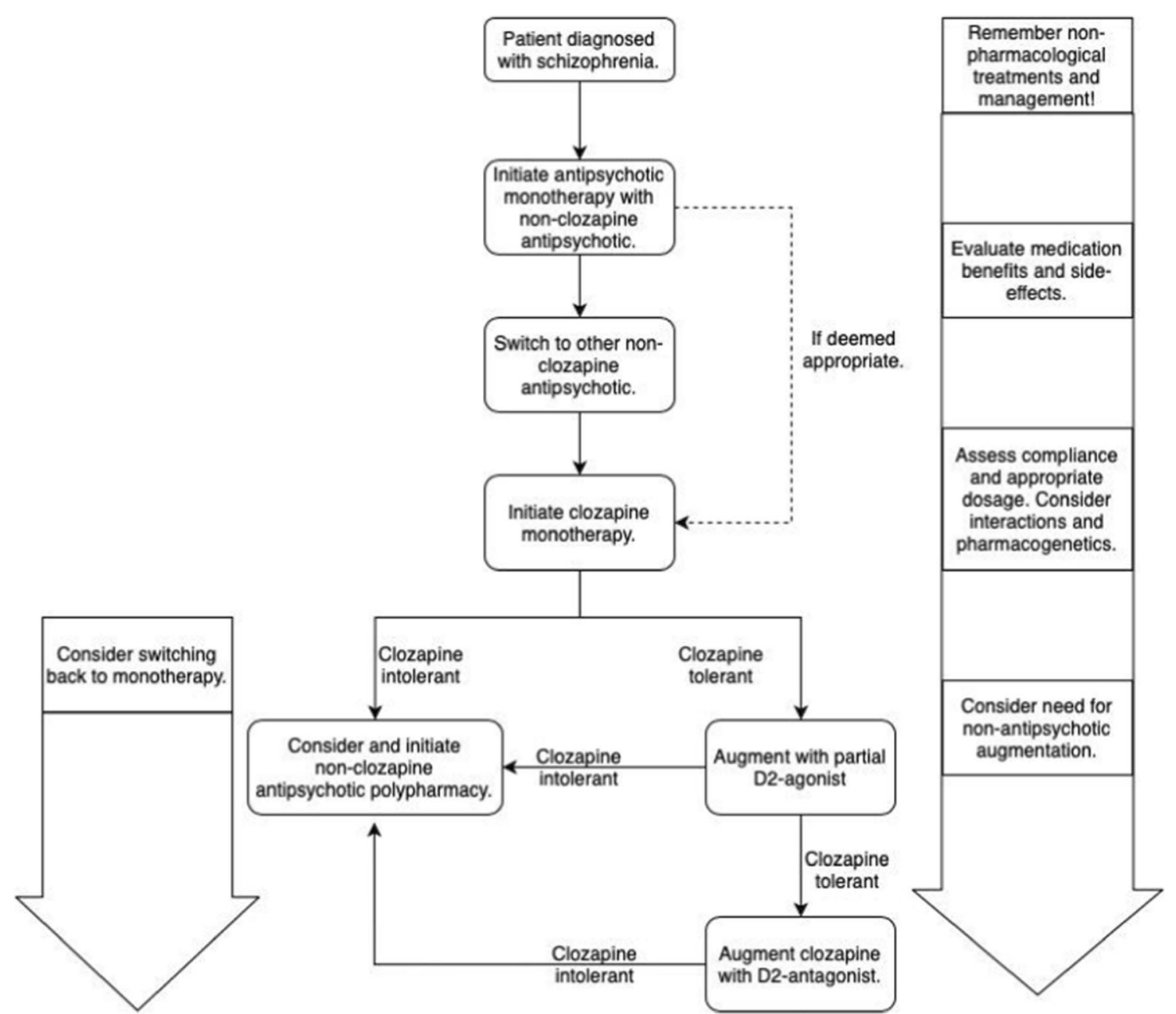

Fig. 1 Flowchart of antipsychotic treatment progression. Progress downwards on the flowchart if disturbing residual symptoms exist 
some cases, APP may produce better results than other forms of treatment augmentation, such as benzodiazepines. In particular, combining aripiprazole with clozapine may be effective in reducing the clozapine dose needed, treatment side effects, and residual symptoms and this is likely to hold true for combining other partial $\mathrm{D}_{2}$ agonists with clozapine as well, although currently not much data exist. Antipsychotic polypharmacy seems safe and may be more effective than monotherapy for some patient groups. However, a clozapine trial should always be seriously considered before switching to APP, as clozapine has been shown to be the most effective treatment for treatment-resistant patients. When used at proper doses and with safety precautions, clozapine is safe and usually well tolerated. More research is needed, both in controlled and real-world settings, to determine optimal APP and/or other psychotropic treatment augmentation strategies for specific patient groups and situations. However, in several studies, many patients treated with APP tolerated well the transition back to monotherapy, indicating that APP may only be needed for some patients at times of symptom exacerbations, and that the use of APP may be in general too widespread or long lasting. It is also essential to remember that as schizophrenia is a multi-etiological disorder, some patients with psychosis may wish to be treated without antipsychotic medications or may not respond to them at all and should be cared for with other approaches [20, 104].

Additionally, treatment guidelines should take greater care to report what is known and what is not, and not make definitive statements on the basis of what is not known or has not been properly studied. It is not the same to state that patients should always be treated with APM, as to state that the evidence base for the effectiveness or safety of APP is insufficient to make definitive recommendations on whether to use it or not. Let us wait for the evidence before we rule out APP from everyone. The existing evidence seems to indicate that APP might have its uses, although it is not appropriate for all patients.

\section{Declarations}

Funding Open access funding provided by University of Eastern Finland (UEF) including Kuopio University Hospital. This work was supported by the Finnish Ministry of Social Affairs and Health through the developmental fund for Niuvanniemi Hospital. Markku Lähteenvuo has also received personal research funding from The Finnish Medical Foundation for the period of this work.

Conflicts of interest Jari Tiihonen reports personal fees from the Finnish Medicines Agency (Fimea), European Medicines Agency, Eli Lilly, Janssen-Cilag, Lundbeck, and Otsuka; is a member of an advisory board for Lundbeck; has participated in research projects funded by grants from Janssen-Cilag and Eli Lilly to his employing institution, and has received grants from the Stanley Foundation and Sigrid Jusélius Foundation. Markku Lähteenvuo is a board member of Genomi Solutions Ltd. and Nursie Health Ltd., and has received honoraria from
Sunovion Ltd., Orion Pharma Ltd. Otsuka Ltd., Lundbeck Ltd., and Janssen-Cilag.

Ethics approval Not applicable.

Consent to participate Not applicable.

Consent for publication Not applicable.

Availability of data and material Data sharing is not applicable to this article as no datasets were generated or analyzed during the current study.

Code availability Not applicable.

Authors' contributions This review was requested and instructed by the journal. ML conceptualized and drafted the manuscript. JT planned the work and provided critical revision for important intellectual content.

Open Access This article is licensed under a Creative Commons Attribution-NonCommercial 4.0 International License, which permits any non-commercial use, sharing, adaptation, distribution and reproduction in any medium or format, as long as you give appropriate credit to the original author(s) and the source, provide a link to the Creative Commons licence, and indicate if changes were made. The images or other third party material in this article are included in the article's Creative Commons licence, unless indicated otherwise in a credit line to the material. If material is not included in the article's Creative Commons licence and your intended use is not permitted by statutory regulation or exceeds the permitted use, you will need to obtain permission directly from the copyright holder. To view a copy of this licence, visit http://creativecommons.org/licenses/by-nc/4.0/.

\section{References}

1. Moreno-Küstner B, Martín C, Pastor L. Prevalence of psychotic disorders and its association with methodological issues: a systematic review and meta-analyses. PLoS ONE. 2018;13: e0195687. https://doi.org/10.1371/journal.pone.0195687.

2. Owen MJ, O'Donovan MC. Schizophrenia and the neurodevelopmental continuum: evidence from genomics. World Psychiatry. 2017;16:227-35.

3. Sullivan PF, Kendler KS, Neale MC. Schizophrenia as a complex trait: evidence from a meta-analysis of twin studies. Arch Gen Psychiatry. 2003;60:1187-92.

4. Smeland OB, Frei O, Dale AM, Andreassen OA. The polygenic architecture of schizophrenia: rethinking pathogenesis and nosology. Nat Rev Neurol. 2020;16:366-79.

5. Ripke S, Neale BM, Corvin A, et al. Biological insights from 108 schizophrenia-associated genetic loci. Nature. 2014;511:421-7.

6. Stilo SA, Murray RM. Non-genetic factors in schizophrenia. Curr Psychiatry Rep. 2019;21:100. https://doi.org/10.1007/ s11920-019-1091-3.

7. Gejman PV, Sanders AR, Duan J. The role of genetics in the etiology of schizophrenia. Psychiatr Clin North Am. 2010;3:35-66.

8. Potkin SG, Kane JM, Correll CU, Lindenmayer JP, Agid O, Marder SR, et al. The neurobiology of treatment-resistant schizophrenia: paths to antipsychotic resistance and a roadmap for future research. NPJ Schizophr. 2020;6:1. https://doi.org/10. 1038/s41537-019-0090-Z. 
9. Tiihonen J, Koskuvi M, Storvik M, et al. Sex-specific transcriptional and proteomic signatures in schizophrenia. Nat Commun. 2019;10:3933. https://doi.org/10.1038/s41467-019-11797-3.

10. McCutcheon RA, Krystal JH, Howes OD. Dopamine and glutamate in schizophrenia: biology, symptoms and treatment. World Psychiatry. 2020;19:15-33.

11. Sommer IE, Bearden CE, van Dellen E, et al. Early interventions in risk groups for schizophrenia: what are we waiting for? NPJ Schizophr. 2016;2:16003. https://doi.org/10.1038/npjschz. 2016.3.

12. Kurachi M, Takahashi T, Sumiyoshi T, Uehara T, Suzuki M. Early intervention and a direction of novel therapeutics for the improvement of functional outcomes in schizophrenia: a selective review. Front Psychiatry. 2018;9:39. https://doi.org/ 10.3389/fpsyt.2018.00039.

13. Keepers GA, Fochtmann LJ, Anzia JM, et al. The American Psychiatric Association practice guideline for the treatment of patients with schizophrenia. Am J Psychiatry. 2020;177:868-72.

14. Kahn RS, Sommer IE, Murray RM, et al. Schizophrenia. Nat Rev Dis Primers. 2015;1:15067. https://doi.org/10.1038/nrdp. 2015. 67.

15. Owen MJ, Sawa A, Mortensen PB. Schizophrenia. Lancet. 2016;388:86-97.

16. Kay SR, Fiszbein A, Opler LA. The Positive and Negative Syndrome Scale (PANSS) for schizophrenia. Schizophr Bull. 1987;13:261-76.

17. Schwieler L, Engberg G, Erhardt S. Clozapine modulates midbrain dopamine neuron firing via interaction with the NMDA receptor complex. Synapse. 2004;52:114-22.

18. Farooq S, Agid O, Foussias G, Remington G. Using treatment response to subtype schizophrenia: proposal for a new paradigm in classification. Schizophr Bull. 2013;39:1169-72.

19. Siskind D, Siskind V, Kisely S. Clozapine response rates among people with treatment-resistant schizophrenia: data from a systematic review and meta-analysis. Can J Psychiatry. 2017;62:772-7.

20. Wils RS, Gotfredsen DR, Hjorth $ø$ j C, Austin SF, Albert N, Secher RG, et al. Antipsychotic medication and remission of psychotic symptoms 10 years after a first-episode psychosis. Schizophr Res. 2017;182:42-8.

21. Jeppesen R, Benros ME. Autoimmune diseases and psychotic disorders. Front Psychiatry. 2019;10:131. https://doi.org/10. 3389/fpsyt.2019.00131.

22. Leza JC, Bueno B, Bioque M, Arango C, Parellada M, Do K, et al. Inflammation in schizophrenia: a question of balance. Neurosci Biobehav Rev. 2015;55:612-26.

23. Müller N, Weidinger E, Leitner B, Schwarz MJ. The role of inflammation in schizophrenia. Front Neurosci. 2015;9:372. https://doi.org/10.3389/fnins.2015.00372.

24. Correll CU, Rummel-Kluge C, Corves C, Kane JM, Leucht S. Antipsychotic combinations vs monotherapy in schizophrenia: a meta-analysis of randomized controlled trials. Schizophr Bull. 2009;35:443-57.

25. Barbui C, Signoretti A, Mule S, Boso M, Cipriani A. Does the addition of a second antipsychotic drug improve clozapine treatment? Schizophr Bull. 2009;35:458-68.

26. Taylor DM, Smith L, Gee SH, Nielsen J. Augmentation of clozapine with a second antipsychotic: a meta-analysis. Acta Psychiatr Scand. 2012;125:15-24.

27. Sommer IE, Begemann MJH, Temmerman A, Leucht S. Pharmacological augmentation strategies for schizophrenia patients with insufficient response to clozapine: a quantitative literature review. Schizophr Bull. 2012;8:1003-11.

28 Galling B, Roldán A, Hagi K, et al. Antipsychotic augmentation vs. monotherapy in schizophrenia: systematic review, meta-analysis and meta-regression analysis. World Psychiatry. 2017;16:77-89.

29. Gallego JA, Nielsen J, de Hert M, Kane JM, Correll CU. Safety and tolerability of antipsychotic polypharmacy. Expert Opin Drug Saf. 2012;11:527-42.

30. Ballon J, Stroup TS. Polypharmacy for schizophrenia. Curr Opin Psychiatry. 2013;26:208-13.

31. Baandrup L. Polypharmacy in schizophrenia. Basic Clin Pharmacol Toxicol. 2020;126:183-92.

32. Fleischhacker WW, Uchida H. Critical review of antipsychotic polypharmacy in the treatment of schizophrenia. Int J Neuropsychopharmacol. 2014;17:1083-93.

33. Stahl S, Grady M. A critical review of atypical antipsychotic utilization: comparing monotherapy with polypharmacy and augmentation. Curr Med Chem. 2005;11:313-27.

34. Lin SK. Antipsychotic polypharmacy: a dirty little secret or a fashion? Int J Neuropsychopharmacol. 2021;23:125-31.

35. Tranulis C, Skalli L, Nicole L, Stip E. Benefits and risks of antipsychotic polypharmacy: an evidence-based review of the literature. Drug Saf. 2008;31:7-20.

36. Taylor DM, Smith L. Augmentation of clozapine with a second antipsychotic: a meta-analysis of randomized, placebo-controlled studies. Acta Psychiatr Scand. 2009;119:419-25.

37. Paton C, Whittington C, Barnes TR. Augmentation with a second antipsychotic in patients with schizophrenia who partially respond to clozapine: a meta-analysis. J Clin Psychopharmacol. 2007;27:198-204.

38. Kennedy-Martin T, Curtis S, Faries D, Robinson S, Johnston $\mathrm{J}$. A literature review on the representativeness of randomized controlled trial samples and implications for the external validity of trial results. Trials. 2015;16:495. https://doi.org/10.1186/ s13063-015-1023-4.

39. Wong JJ, Jones N, Timko C, Humphreys K. Exclusion criteria and generalizability in bipolar disorder treatment trials. Contemp Clin Trials Commun. 2018;9:130-4.

40. Nordon C, Bovagnet T, Belger M, Jimenez J, Olivares R, Chevrou-Severac $\mathrm{H}$, et al. Trial exclusion criteria and their impact on the estimation of antipsychotic drugs effect: a case study using the SOHO database. Schizophr Res. 2018;193:146-53.

41. Correll CU. Randomized controlled trials in schizophrenia: opportunities, limitations, and trial design alternatives. Dialogues Clin Neurosci. 2011;13:155-72.

42. McDonagh MS, Dana T, Selph S, Devine EB, Cantor A, Bougatsos C, et al. Treatments for schizophrenia in adults: a systematic review. Rockville (MD): Agency for Healthcare Research and Quality (US); 2017: Report No.: 17(18)-EHC031-EF. 2017.

43. NCCMH. Psychosis and schizophrenia in adults: treatment and management. In: London: NICE; 2014: Clinical Guideline No. 178. 2014.

44. Hasan A, Falkai P, Wobrock T, et al. World Federation of Societies of Biological Psychiatry (WFSBP) guidelines for biological treatment of schizophrenia, part 1: update 2012 on the acute treatment of schizophrenia and the management of treatment resistance. World J Biol Psychiatry. 2012;13:318-78.

45. Finnish W Group set up by the FMSD and the FP Association. Schizophrenia: current care guidelines. 2020. https://www.kaypa hoito.fi/hoi35050. Accessed 3 Dec 2020.

46. Gallego JA, Bonetti J, Zhang J, Kane JM. Prevalence and correlates of antipsychotic polypharmacy: a systematic review and meta-regression of global and regional trends from the 1970s to 2009. Schizophr Res. 2012;138:18-28.

47. Dong M, Zeng LN, Zhang Q, et al. Prescription of antipsychotic and concomitant medications for adult Asian schizophrenia patients: findings of the 2016 Research on Asian Psychotropic Prescription Patterns (REAP) survey. Asian J Psychiatry. 2019;45:74-80. 
48. Tiihonen J, Taipale H, Mehtälä J, Vattulainen P, Correll CU, Tanskanen A. Association of antipsychotic polypharmacy vs monotherapy with psychiatric rehospitalization among adults with schizophrenia. JAMA Psychiat. 2019;76:499-507.

49. Yazici E, Cilli AS, Yazici AB, Baysan H, Ince M, Bosgelmez S, et al. Antipsychotic use pattern in schizophrenia outpatients: correlates of polypharmacy. Clin Pract Epidemiol Ment Health. 2017;13:92-103.

50. Sernyak MJ, Rosenheck R. Clinicians' reasons for antipsychotic coprescribing. J Clin Psychiatry. 2004;65:1597-600.

51. Lin CH, Wang FC, Lin SC, Huang YH, Chen CC, Lane HY. Antipsychotic combination using low-dose antipsychotics is as efficacious and safe as, but cheaper, than optimal-dose monotherapy in the treatment of schizophrenia: a randomized, double-blind study. Int Clin Psychopharmacol. 2013;28:267-74.

52. Stummer L, Markovic M, Maroney M. Pharmacologic treatment options for insomnia in patients with schizophrenia. Medicines. 2018;5:88

53 Dodds TJ. Prescribed benzodiazepines and suicide risk: a review of the literature. Prim Care Companion CNS Disord. 2017;2017:19. https://doi.org/10.4088/PCC.16r02037.

54. Barker MJ, Greenwood KM, Jackson M, Crowe SF. Cognitive effects of long-term benzodiazepine use: a meta-analysis. CNS Drugs. 2004;18:37-48.

55. Soyka M. Treatment of benzodiazepine dependence. N Engl J Med. 2017;376:1147-57.

56. Kadra G, Stewart R, Shetty H, Downs J, MacCabe JH, Taylor $\mathrm{D}$, et al. Predictors of long-term ( $\geq 6$ months) antipsychotic polypharmacy prescribing in secondary mental healthcare. Schizophr Res. 2016;174:106-12.

57. Barbui C, Nosè M, Mazzi MA, et al. Persistence with polypharmacy and excessive dosing in patients with schizophrenia treated in four European countries. Int Clin Psychopharmacol. 2006;21:355-62.

58. Kreyenbuhl JA, Valenstein M, McCarthy JF, Ganoczy D, Blow FC. Long-term antipsychotic polypharmacy in the VA health system: patient characteristics and treatment patterns. Psychiatr Serv. 2007;58:489-95.

59. Correll CU, Rubio JM, Inczedy-Farkas G, Birnbaum ML, Kane JM, Leucht S. Efficacy of 42 pharmacologic cotreatment strategies added to antipsychotic monotherapy in schizophrenia: systematic overview and quality appraisal of the meta-analytic evidence. JAMA Psychiat. 2017;74:675-84.

60 Matsui K, Tokumasu T, Takekita Y, et al. Switching to antipsychotic monotherapy vs staying on antipsychotic polypharmacy in schizophrenia: a systematic review and meta-analysis. Schizophr Res. 2019;209:50-7.

61. Baandrup L. How dosing might influence the conclusion in an antipsychotic polypharmacy effectiveness trial. Am J Psychiatry. 2011;168:1117.

62. Constantine RJ, Andel R, McPherson M, Tandon R. The risks and benefits of switching patients with schizophrenia or schizoaffective disorder from two to one antipsychotic medication: a randomized controlled trial. Schizophr Res. 2015;166:194-200.

63. Constantine RJ, Andel R, McPherson M, Tandon R. Is the risk of antipsychotic polypharmacy discontinuation dependent on the agents used? Psychiatry Res. 2018;263:238-44.

64 Katona L, Czobor P, Bitter I. Real-world effectiveness of antipsychotic monotherapy vs. polypharmacy in schizophrenia: to switch or to combine? A nationwide study in Hungary. Schizophr Res. 2014;152:246-54.

65. Hatta K, Hasegawa H, Imai A, et al. Real-world effectiveness of antipsychotic monotherapy and polytherapy in 1543 patients with acute-phase schizophrenia. Asian J Psychiatry. 2019;40:82-7.
66. Taipale H, Tanskanen A, Mehtälä J, Vattulainen P, Correll CU, Tiihonen J. 20-year follow-up study of physical morbidity and mortality in relationship to antipsychotic treatment in a nationwide cohort of 62,250 patients with schizophrenia (FIN20). World Psychiatry. 2020;19:61-8.

67. Tiihonena J, Suokas JT, Suvisaari JM, Haukka J, Korhonen P. Polypharmacy with antipsychotics, antidepressants, or benzodiazepines and mortality in schizophrenia. Arch Gen Psychiatry. 2012;69:476-83.

68. Tiihonen J, Mittendorfer-Rutz E, Majak M, et al. Real-world effectiveness of antipsychotic treatments in a nationwide cohort of 29823 patients with schizophrenia. JAMA Psychiat. 2017;74:686-93.

69. Baandrup L, Gasse C, Jensen VD, Glenthoj BY, Nordentoft $\mathrm{M}$, Lublin $\mathrm{H}$, et al. Antipsychotic polypharmacy and risk of death from natural causes in patients with schizophrenia: a population-based nested case-control study. J Clin Psychiatry. 2010;71:103-8.

70. Kadra G, Stewart R, Shetty H, MacCabe JH, Chang CK, Taylor $\mathrm{D}$, et al. Long-term antipsychotic polypharmacy prescribing in secondary mental health care and the risk of mortality. Acta Psychiatr Scand. 2018;138:123-32.

71. Kasteridis P, Ride J, Gutacker N, et al. Association between antipsychotic polypharmacy and outcomes for people with serious mental illness in England. Psychiatr Serv. 2019;70:650-6.

72. Chen Y, Yang X, Qin X, et al. Antipsychotics and risk of natural death in patients with schizophrenia. Neuropsychiatric Dis Treat. 2019;15:1863-71.

73. Barbui C, Biancosino B, Esposito E, Marmai L, Donà S, Grassi L. Factors associated with antipsychotic dosing in psychiatric inpatients: a prospective study. Int Clin Psychopharmacol. 2007;22:221-5.

74. Centorrino F, Goren JL, Hennen J, Salvatore P, Kelleher JP, Baldessarini RJ. Multiple versus single antipsychotic agents for hospitalized psychiatric patients: case-control study of risks versus benefits. Am J Psychiatry. 2004;161:700-6.

75. Kreyenbuhl J, Valenstein M, McCarthy JF, Ganoczy D, Blow FC. Long-term combination antipsychotic treatment in VA patients with schizophrenia. Schizophr Res. 2006;84:90-9.

76. Kadra G, Stewart R, Shetty H, MacCabe JH, Chang CK, Kesserwani J, et al. Antipsychotic polypharmacy prescribing and risk of hospital readmission. Psychopharmacology. 2018;235:281-9.

77. Paulzen M, Schoretsanitis G, Stegmann B, Hiemke C, Gründer $\mathrm{G}$, Schruers KRJ, et al. Pharmacokinetic considerations in antipsychotic augmentation strategies: how to combine risperidone with low-potency antipsychotics. Prog Neuropsychopharmacol Biol Psychiatry. 2017;76:101-6.

78. Schoretsanitis G, Haen E, Hiemke C, Grönder G, Stegmannb $\mathrm{B}$, Schruers KRJ, et al. Risperidone-induced extrapyramidal side effects: Is the need for anticholinergics the consequence of high plasma concentrations? Int Clin Psychopharmacol. 2016;31:259-64.

79. Paulzen M, Haen E, Hiemke C, Stegmann B, Lammertz SE, Gründer G, Schoretsanitis G. Cytochrome P450-mediated interaction between perazine and risperidone: implications for antipsychotic polypharmacy. Br J Clin Pharmacol. 2017;83:1668-75.

80. Élie D, Poirier M, Chianetta JM, Durand M, Grégoire CA, Grignon S. Cognitive effects of antipsychotic dosage and polypharmacy: a study with the BACS in patients with schizophrenia and schizoaffective disorder. J Psychopharmacol. 2010;24:1037-44.

81. Kontis D, Theochari E, Kleisas S, Kalogerakou S, Andreopoulou A, Psaras R, et al. Doubtful association of antipsychotic polypharmacy and high dosage with cognition in chronic 
schizophrenia. Prog Neuropsychopharmacol Biol Psychiatry. 2010;34:1333-41.

82. Baandrup L, Sørensen J, Lublin H, Nordentoft M, Glenthoj B. Association of antipsychotic polypharmacy with health service cost: a register-based cost analysis. Eur J Health Econ. 2012;13:355-63.

83. Kane JM, Kishimoto T, Correll CU. Non-adherence to medication in patients with psychotic disorders: epidemiology, contributing factors and management strategies. World Psychiatry. 2013;12:216-26.

84. Narasimhan M, Pae CU, Masand N, Masand P. Partial compliance with antipsychotics and its impact on patient outcomes. Int J Psychiatry Clin Pract. 2007;11:102-11.

85. Masand PS, Roca M, Turner MS, Kane JM. Partial adherence to antipsychotic medication impacts the course of illness in patients with schizophrenia: a review. Prim Care Companion J Clin Psychiatry. 2009;11:147-54.

86. van Westrhenen R, Aitchison KJ, Ingelman-Sundberg M, Jukić MM. Pharmacogenomics of antidepressant and antipsychotic treatment: how far have we got and where are we going? Front Psychiatry. 2020;11:94. https://doi.org/10.3389/fpsyt. 2020. 00094.

87. Jukic MM, Smith RL, Haslemo T, Molden E, Ingelman-Sundberg M. Effect of CYP2D6 genotype on exposure and efficacy of risperidone and aripiprazole: a retrospective, cohort study. Lancet Psychiatry. 2019;6:418-26.

88. Hunt GE, Bergen J, Bashir M. Medication compliance and comorbid substance abuse in schizophrenia: impact on community survival 4 years after a relapse. Schizophr Res. 2002;54:253-64.

89. Haddad P, Brain C, Scott J. Nonadherence with antipsychotic medication in schizophrenia: challenges and management strategies. Patient Relat Outcome Meas. 2014;5:43-62.

90. Taipale H, Mehtälä J, Tanskanen A, Tiihonen J. Comparative effectiveness of antipsychotic drugs for rehospitalization in schizophrenia: a nationwide study with 20 -year follow-up. Schizophr Bull. 2018;44:1381-7.

91. Bachmann CJ, Aagaard L, Bernardo M, et al. International trends in clozapine use: a study in 17 countries. Acta Psychiatr Scand. 2017;136:37-51.

92. Agid O, Arenovich T, Sajeev G, Zipursky RB, Kapur S, Foussias $\mathrm{G}$, et al. An algorithm-based approach to first-episode schizophrenia: response rates over 3 prospective antipsychotic trials with a retrospective data analysis. J Clin Psychiatry. 2011;72:1439-44.

93. Okhuijsen-Pfeifer C, Huijsman EAH, Hasan A, Sommer IEC, Leucht S, Kahn RS, et al. Clozapine as a first- or second-line treatment in schizophrenia: a systematic review and meta-analysis. Acta Psychiatr Scand. 2018;138:281-8.

94 Kahn RS, van Winter Rossum I, Leucht S, et al. Amisulpride and olanzapine followed by open-label treatment with clozapine in first-episode schizophrenia and schizophreniform disorder (OPTiMiSE): a three-phase switching study. Lancet Psychiatry. 2018;5:797-807.

95. Srisurapanont M, Suttajit S, Maneeton N, Maneeton B. Efficacy and safety of aripiprazole augmentation of clozapine in schizophrenia: a systematic review and meta-analysis of randomizedcontrolled trials. J Psychiatr Res. 2015;62:38-47.

96. Chang JS, Ahn YM, Park HJ, Lee KY, Kim SH, Kang UG, et al. Aripiprazole augmentation in clozapine-treated patients with refractory schizophrenia: an 8-week, randomized, double-blind, placebo-controlled trial. J Clin Psychiatry. 2008;69:720-31.

97. Muscatello MRA, Bruno A, Pandolfo G, Micò U, Scimeca G, di Nardo F, et al. Effect of aripiprazole augmentation of clozapine in schizophrenia: a double-blind, placebo-controlled study. Schizophr Res. 2011;127:93-9.

98. Arranz MJ, Gonzalez-Rodriguez A, Perez-Blanco J, et al. A pharmacogenetic intervention for the improvement of the safety profile of antipsychotic treatments. Transl Psychiatry. 2019;9:177. https://doi.org/10.1038/s41398-019-0511-9.

99. Malhotra AK, Murphy GM, Kennedy JL. Pharmacogenetics of psychotropic drug response. Am J Psychiatry. 2004;161:780-96.

100. Zhang JP, Lencz T, Zhang RX, et al. Pharmacogenetic associations of antipsychotic drug-related weight gain: a systematic review and meta-analysis. Schizophrenia Bull. 2016;42:1418-37.

101. Lally J, Gaughran F, Timms P, Curran SR. Treatment-resistant schizophrenia: current insights on the pharmacogenomics of antipsychotics. Pharmacogenomics Pers Med. 2016;9:117-29.

102. Essock SM, Schooler NR, Stroup TS, et al. Effectiveness of switching from antipsychotic polypharmacy to monotherapy. Am J Psychiatry. 2011;168:702-8.

103. Borlido C, Remington G, Graff-Guerrero A, Arenovich T, Hazra $\mathrm{M}$, Wong A, et al. Switching from 2 antipsychotics to 1 antipsychotic in schizophrenia: a randomized, double-blind, placebocontrolled study. J Clin Psychiatry. 2016;77:e14-20.

104. Wunderink L, Nieboer RM, Wiersma D, Sytema S, Nienhuis FJ. Recovery in remitted first-episode psychosis at 7 years of followup of an early dose reduction/discontinuation or maintenance treatment strategy long-term follow-up of a 2-year randomized clinical trial. JAMA Psychiat. 2013;70:913-20. 